\title{
A Class of Generalized Median Contour Problem with Exact Solution
}

\author{
Pakaket Wattuya and Xiaoyi Jiang \\ Department of Mathematics and Computer Science \\ University of Münster, Germany \\ \{wattuya, xjiang\}@math.uni-muenster.de
}

\begin{abstract}
The ability to find the average of a set of contours has several applications in computer vision including prototype formation and computational atlases. While contour averaging can be handled in an informal manner, the formal formulation within the framework of generalized median as an optimization problem is attractive. In this work we will follow this line. A special class of contours is considered, which start from the top, pass each image row exactly once, and end in the last row of an image. Despite of the simplicity they frequently occur in many applications of image analysis. We propose a dynamic programming approach to exactly compute the generalized median contour in this domain. Experimental results will be reported on two scenarios to demonstrate the usefulness of the concept of generalized median contours. In the first case we postulate a general approach to implicitly explore the parameter space of a (segmentation) algorithm. It is shown that using the generalized median contour, we are able to achieve contour detection results comparable to those from explicitly training the parameters based on known ground truth. As another application we apply the exact median contour to verify the tightness of a lower bound for generalized median problems in metric space.
\end{abstract}

\section{Introduction}

The ability to find the average of a set of contours has several applications in computer vision including prototype formation and computational atlases. While contour averaging can be handled in an informal manner as done in [1]1, the formal formulation within the framework of generalized median as an optimization problem is attractive. In this work we will follow this line.

Given a set of $n$ patterns $C_{1}, C_{2}, \ldots, C_{n}$ in an arbitrary representation space $U$, we assume a distance function $d(p, q)$ to measure the dissimilarity between any two patterns $p, q \in U$. Then, the generalized median $\bar{C}$ is defined by:

$$
\bar{C}=\arg \min _{C \in U} \sum_{i=1}^{n} d\left(C, C_{i}\right)
$$

This concept has been successfully applied to strings [7/9] and graphs [5] in structured pattern recognition. 
If a contour is coded by a string, then the same procedure can be adapted to averaging contours [7. However, this general approach suffers from high computational complexity. It is proved in 4 that computing the generalized median string is NP-hard. Sim and Park [12] proved that the problem is NP-hard for finite alphabet and for a metric distance matrix. Another result comes from computational biology. The optimal evolutionary tree problem there turns out to be equivalent to the problem of computing generalized median strings if the tree structure is a star (a tree with $n+1$ nodes, $n$ of them being leaves). In 13 . it is proved that in this particular case the optimal evolutionary tree problem is NP-hard. The distance function used is problem dependent and does not even satisfy the triangle inequality. All these theoretical results indicate the inherent difficulty in finding generalized median strings, or equivalently the generalized median contours. Not surprisingly, researchers make use of domain-specific knowledge to reduce the complexity [9] or resort to approximate approaches [7.

In this work we consider a special class of contours for which the generalized median can be found by an efficient algorithm based on dynamic programming. We first motivate our work by giving some background information about this class of contours. Then, the algorithm for finding the exact solution is described in Section 3. In Section 4 we describe two applications of generalized median computation: exploring the parameter space of a contour detection algorithm and tightness evaluation of a lower bound of generalized median problems in metric space. Finally, some discussion conclude the paper.

\section{Class of Contours}

The class of contours considered in this work is defined as follows:

Definition 1. For a given $M \times N$ image a contour $C=p_{1} p_{2}, \ldots, p_{M}$ is a sequence of points drawn from the top to the bottom, where $p_{i}, i=1, \ldots, M$, is a point in the $i$-th row. The points $p_{i}$ and $p_{i+1}, i=1, \ldots, M-1$, of two successive rows are continuous.

These contours start from the top, pass each image row exactly once, and end in the last row.

At the first glance the question may arise why such simple contours are of use in practice. Some thoughts, however, reveal that there do exist several situations, where we are directly or indirectly faced with this class of contours. In medical imaging it is typical for the user to specify some region of interest (ROI) and then to find some contours within the ROI. As an example, Figure 1 shows
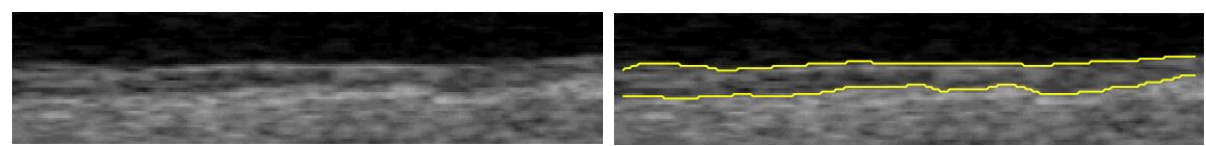

Fig. 1. ROI in a CCA B-mode sonographic image (left) and detected layer of intima and adventitia (right) 


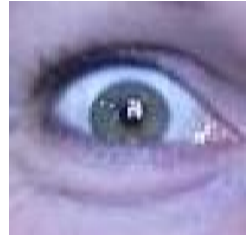

(a)

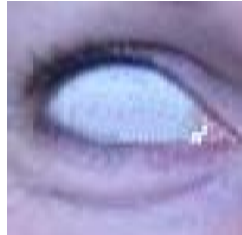

(b)

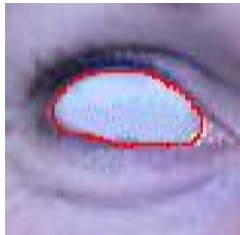

(c)

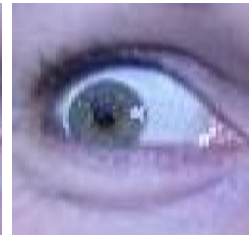

(d)

Fig. 2. Detection of closed contour: (a) input image; (b) removal of iris; (c) detection of eye contour; (d) strabismus simulation

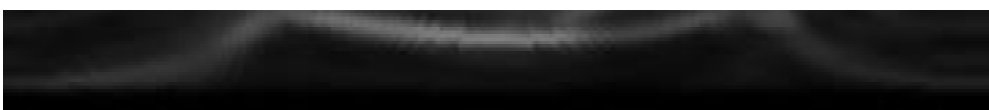

(a)

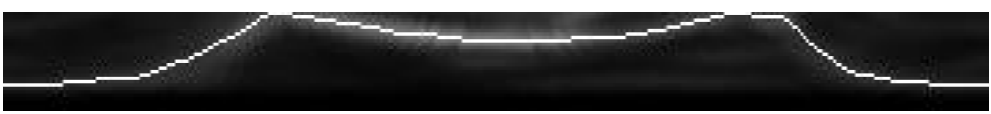

(b)

Fig. 3. Polar space for contour detection: (a) polar space; (b) optimal path.

a ROI in a CCA (Common Carotid Artery) B-mode sonographic image. The task is to detect the layer of intima and adventitia for computing the intimamedia thickness which is an important index in modern medicine. Details of this application and an algorithm for automatic layer detection can be found in [2]. Essential to the current work is the fact that both the intimal layer and the adventitial layer are examples of the contour class defined above (although we have to rotate the image by 90 degrees). This application reflects a typical situation in medical image analysis.

The same fundamental principle can be extended to deal with closed contours. For this purpose we need a point $p$ in the interior of the contour. Then, a polar transformation with $p$ being the central point brings the original image into a matrix, in which a closed contour becomes a contour from top to bottom afterwards. Note that this technique works well for all star-shaped contours including convex contours as a special case. As an example, Figure 2 shows a problem of eye contour detection taken from [8]. In the image after removal of iris, the eye contour is detected as a closed contour based on the interior reflection point. The polar space representation related to Figure 2(b) can be seen in Figure 3(a) where the intensity is replaced by a measure of edge magnitude. In this space we are faced with the same contour detection problem as in Figure 1. The result is shown in Figure 3(b) and Figure 2(c) after projecting back into the image space. The task in this application is then to simulate strabismus by replacing the iris. The eye contour serves to restrict the region, within which the newly positioned iris lies. For (almost) convex contours the selection of the origin of polar space is not critical. In the general case of star-shaped contours, 
however, it must be chosen within the area, in which the complete contour can be seen.

The two situations above and others appear in a variety of applications. They indicate the broad applicability of the class of contours considered in this paper and thus justify to investigate them in their own right.

The concept of generalized median in Eqn. (1) can be easily adapted to our domain by specifying a distance function between two contours. Since each point $p_{i}$ of a contour $P=p_{1} p_{2}, \ldots, p_{M}$ has a constant $y$-coordinate $i$, we use $p_{i}$ to represent its $x$-coordinate only in the following in order to simplify the notation. Given this convention, the distance between two contours $P$ and $Q$ can be defined by the $k$-th power of the Minkowski distance:

$$
d(P, Q)=\sum_{i=1}^{M}\left(p_{i}-q_{i}\right)^{k}
$$

In this case the representation space $U$ contains all continuous contours from top to bottom of an input $M \times N$ image.

\section{Computation of Generalized Median Contours}

Given $n$ contours $C_{1}, C_{2}, \ldots, C_{n}$, the task is to determine a contour $\bar{C}$ such that the sum of distances between $\bar{C}$ and all input contours is minimized. It is important to notice that we cannot solve this problem of generalized median contours by computing the optimal value for each of the $M$ rows independently, which could be done, for instance, by enumerating all possibilities between the leftmost and rightmost point in the row. Doing it this way, we encounter the trouble of generating a discontinuous resultant contour.

Our proposed method is formulated as a problem of finding an optimal path in a graph based on dynamic programming. We first generate a two-dimensional $M \times N$ cost matrix of the same size as the image, in which every element corresponds to an image point. Each element is assigned a Local_Goodness value, which measures its suitability of being a candidate point on the generalized median contour we are looking for. According to the distance given in Eqn. (2) the Local_Goodness value is simply:

$$
\operatorname{Local}_{\text {Goodness }}(i, j)=\sum_{l=1}^{n}\left(x_{l i}-j\right)^{k}, \quad 1 \leq i \leq M, 1 \leq j \leq N
$$

where $x_{l i}$ represents the $x$-coordinate of the $l$-th contour $C_{l}$ in $i$-th row. Generally, small Local_Goodness values indicate better candidates. As a matter of fact, the optimality of a candidate for $\bar{C}$ is measured by the sum of its Local_Goodness values over all image rows.

Dynamic programming is applied to search for an optimal path in a cumulative cost matrix $C C$. The cumulative cost of a node $(i, j)$ is computed as:

$$
C C(i, j)=\min _{l=-1,0,1}\{C C(i-1, j+l)\}+\text { Local_Goodness }(i, j)
$$


for $2 \leq i \leq M, 1 \leq j \leq N$. This means that a contour point $(i, j)$ has three potential predecessors $(i-1, j-1),(i-1, j),(i-1, j+1)$ in the previous row. In addition, the choice of a transition from a point in $i$-th row to a predecessor in the $(i-1)$-th row is made based on the lowest cumulative cost of the predecessors. The computation of $C C$ starts by initializing the first row by:

$$
C C(1, j)=\text { Local_Goodness }(1, j), \quad 1 \leq j \leq N
$$

Then, the cumulative cost matrix $C C$ is filled row by row from left to right by using Eqn. (3).

The node in the last row of matrix $C C$ with the lowest value gives us the last point of the optimum path. To determine this path, a matrix of pointers is created at the time of computing the matrix CC. The optimum path, which corresponds to the generalized median contour, is determined by starting at the last point and following the pointers back to the first row. Using this dynamic programming technique, we are able to compute the generalized median contour exactly.

The computational complexity of the algorithm amounts to $O(M N n)$ while $O(M N)$ space is required. Note that the search space of dynamic programming can be substantially reduced. For each row we only need to consider the range bounded by the leftmost and rightmost point from all input contours in that row. The size of this reduced search space depends on the variation of input data. The less variation of the input data, the more the reduction effect. Most likely, this reduction results in a computational complexity of $O(M n)$ only. The proposed algorithm was implemented in Matlab on a Pentium IV $2.1 \mathrm{GHz}$ PC. As an example, the computation time for 250 input contours of 105 points each with 0.00 standard deviation in the input data is 10 milliseconds. At an increased level of data variation of 81.74 standard deviation, 90 milliseconds were recorded. We can conclude that the dynamic programming approach delivers an efficient way of exactly computing the generalized median of contours.

\section{Experimental Results}

We have conducted a series of experiments using both synthetic and real data. In the following we report some results to illustrate two applications of the concept of generalized median contours.

\subsection{Test Images and Contour Data}

Both studies are based on CCA B-mode sonographic images 2. An image dataset was established which consists of 23 such images of 105 columns each. They are actually ROI cut out of larger images. Each image contains two contours of interest: intima $\left(y_{1}\right)$ and adventitia $\left(y_{2}\right)$. Both contours run from left to right of an image. If we turn the images by 90 degrees, then we are faced with the problem of optimally masking the two contours of length 105 each from top to bottom. 
Table 1. Performance measures of parameter training and generalized median (GM) approaches on 5 test sets

\begin{tabular}{ccccc}
\hline & \multicolumn{2}{c}{$y_{1}$ (intima) } & \multicolumn{2}{c}{$y_{2}$ (adventitia) } \\
\cline { 2 - 5 } Test set & Parameter training & GM & Parameter training & GM \\
\hline 1 & 48.98 & 49.77 & 60.59 & 50.18 \\
2 & 48.68 & 49.37 & 53.56 & 52.82 \\
3 & 51.09 & 51.16 & 51.79 & 51.26 \\
4 & 49.90 & 50.66 & 46.83 & 47.08 \\
5 & 46.53 & 46.53 & 50.03 & 48.07 \\
\hline average & 49.04 & 49.50 & 52.56 & 49.88 \\
\hline
\end{tabular}

Each image has its ground truth contours manually specified by an experienced physician. This information is used for an objective, quantitative comparison with automatic detection results. The similarity measure is simply the distance function in Eqn. (2). In all our tests we have fixed $k$ of the distance function to $k=1$.

\subsection{Exploring Parameter Space Without Ground Truth}

Segmentation algorithms mostly have some parameters and their optimal setting is not a trivial task. In recent years automatic parameter training has become popular. Typically, a training image set with (manual) ground truth segmentation is assumed to be available. Then, a subspace of the parameter space is explored to find out the best parameter setting. For each parameter setting candidate a performance measure is computed in the following way:

- Segment each image of the training set based on the parameter setting;

- Compute a performance measure by comparing the segmentation result and the corresponding ground truth;

- Compute the average performance measure over all images of the training set.

The optimal parameter setting is given by the one with the largest average performance measure. Since fully exploring the subspace can be very costly, space subsampling [10, or genetic search [3] has been proposed.

While this approach is reasonable and has been successfully practiced in several applications, its fundamental disadvantage is the assumption of ground truth segmentation. The manual generation of ground truth is always painful and thus a main barrier of wide use in many situations.

We propose to apply the concept of generalized median for implicitly exploring the parameter space without the need of ground truth segmentation. It is assumed that we know a reasonable subspace of the parameter space (i.e. a lower and upper bound for each parameter), which is sampled into a finite number $\mathcal{M}$ of parameter settings. Then, we run the segmentation procedure for all the $\mathcal{M}$ parameter settings and compute the generalized median of the $\mathcal{M}$ segmentation results. The rationale behind our approach is that the median segmentation tends to be a good one within the explored parameter subspace. 
This idea has been verified on the database described above within the contour detection algorithm [2]. It has two parameters and a reasonable parameter subspace is divided into 250 samples. The database is partitioned into a training set of 10 images and a test set of 13 images. The training set is then used to find the optimal parameter setting among the 250 candidates, which is applied to the test set. The average performance measure over the 13 test images is listed in Table 1. Note that the testing procedure is repeated 5 times for different partitions of the 23 images into training and test set. On the other hand, the generalized median approach has no knowledge of the ground truth segmentation. It simply detects 250 contours and computes their generalized median. The average performance measure of the 13 generalized median contours in the test set as shown in Table 1 indicates that basically no real performance differences exist between these two approaches. Without using any ground truth information, the generalized median technique is able to produce contours of essentially identical quality as the training approach.

\section{Verification of Optimal Lower Bound for Generalized Median Problems in Metric Space}

The computation of generalized median patterns is typically an NP-complete task. Therefore, research efforts are focused on approximate approaches. One essential aspect in this context is the assessment of the quality of the computed approximate solutions. Since the true optimum is unknown, the quality assessment is not trivial in general. A recent work [6] presented the lower bound for this purpose.

Referring to the notation in Eqn. (1), an approximate computation method gives us a solution $\tilde{C}$ such that

$$
\operatorname{SOD}(\tilde{C})=\sum_{i=1}^{n} d\left(\tilde{C}, C_{i}\right) \geq \sum_{i=1}^{n} d\left(\bar{C}, C_{i}\right)=\operatorname{SOD}(\bar{C})
$$

where SOD stands for sum of distances and $\bar{C}$ represents the (unknown) true generalized median. The quality of $\tilde{C}$ can be measured by the difference $\operatorname{SOD}(\tilde{C})-$ $\operatorname{SOD}(\bar{C})$. Since $\bar{C}$ and thus $\operatorname{SOD}(\bar{C})$ are unknown in general, we resort to a lower bound $\Gamma \leq \operatorname{SOD}(\bar{C})$ and measure the quality of $\tilde{C}$ by $\operatorname{SOD}(\tilde{C})-\Gamma$. Note that the relationship

$$
0 \leq \Gamma \leq S O D(\bar{C}) \leq S O D(\tilde{C})
$$

holds. Obviously, $\Gamma=0$ is a trivial, and also useless, lower bound. We require $\Gamma$ to be as close to $\operatorname{SOD}(\bar{C})$ as possible. This tightness can be quantified by $\operatorname{SOD}(\bar{C})-\Gamma$ with a value zero for the ideal case.

In [6] the tightness of the lower bound has been tested in the domain of strings and graphs. Since the computation of generalized strings and graphs is exponential, only approximate solutions have been considered there.

Ideally, the tightness should be investigated in domains where we know the true generalized median. The current work provides us a means of validating 

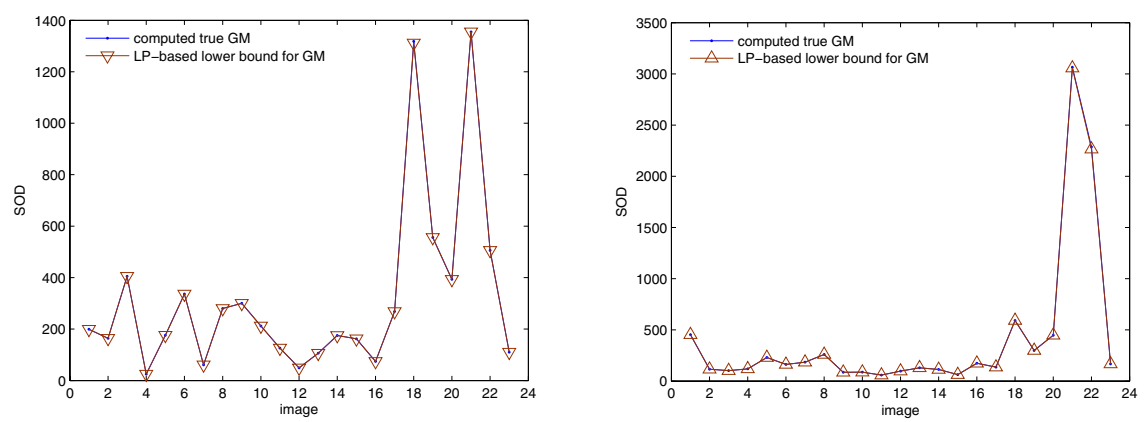

Fig. 4. Tightness of lower bound $\Gamma$ for $50 y_{1}$ contours (intima, left) and $50 y_{2}$ contours (adventitia, right) contours for all 23 images

the tightness under ideal conditions. For this purpose we sampled 50 parameter settings of the parameter subspace 1 . For each image, we thus compute 50 contours and afterwards their exact generalized median $\bar{C}$ by the dynamic programming technique proposed in this paper. In Figure 4 both the lower bound $\Gamma$ and $\operatorname{SOD}(\bar{C})$ for all 23 images are plotted. Obviously, these two values are so similar that no difference is visible. This is clearly a sign of good tightness of the lower bound $\Gamma$. Although this statement is made for the particular case of contours, it builds a piece of the mosaic of validating the tightness in many problem spaces.

\section{Conclusions}

In this paper we have considered a special class of contours which start from the top, pass each image row exactly once, and end in the last row of an image. Despite of the simplicity they frequently occur in many applications of image analysis. We have proposed a dynamic programming approach to exactly compute the generalized median contour in this domain.

Experimental results have been reported on two scenarios, in which the concept of generalized median plays a very different role. In the first case we have postulated a general approach to implicitly explore the parameter space of a (segmentation) algorithm. It was shown that using the generalized median contour, we are able to achieve contour detection results comparable to those from explicitly training the parameters using a training set with known ground truth. This performance is remarkable and should be further investigated in other contexts.

Having a generalized median problem with exact solution is interesting in its own right for the specific problem domain. From a more general point of view,

${ }^{1}$ The reason for selecting only 50 instead of 250 as in other experiments lies in the high computation time and space requirement of the lower bound computation which is based on linear programming. 
the exact solution gives us a means to verify the tightness of the lower bound for generalized median computation under ideal conditions. We have performed the verification which shows the high tightness. As part of our efforts in verifying the tightness of the lower bound using a variety of generalized median problems with exact solution, the current work represents a valuable contribution.

\section{References}

1. V. Chalana and Y. Kim. A methodology for evaluation of boundary detection algorithms on medical images. IEEE Trans. on Medical Imaging, 16(5): 642-652, 1997.

2. D. Cheng, X. Jiang, A. Schmidt-Trucksäss and K. Cheng. Automatic intima-media thickness measurement of carotid artery wall in B-mode sonographic images. Proc. of IEEE International Symposium on Biomedical Imaging: From Nano to Macro, 912-915, 2006.

3. L. Cingue, R. Cucciara, S. Levialdi, S. Martinez, and G. Pignalberi. Optimal range segmentation parameters through genetic algorithms. Proc. of 15th Int. Conf. on Pattern Recognition, Vol. 1, 474-477, Barcelona, 2000.

4. C. de la Higuera and F. Casacuberta. Topology of strings: Median string is NPcomplete. Theoretical Computer Science, 230(1/2): 39-48, 2000.

5. X. Jiang, A. Münger, and H. Bunke. On median graphs: Properties, algorithms, and applications. IEEE Trans. on Pattern Analysis and Machine Intelligence, 23(10): $1144-1151,2001$.

6. X. Jiang and H. Bunke. Optimal lower bound for generalized median problems in metric space. In: Structural, Syntactic, and Statistical Pattern Recognition (T.Caelli, A.Amin, R.P.W.Duin, M.Kamel, and D.de Ridder, Eds.), SpringerVerlag, 143-151, 2002.

7. X. Jiang, K. Abegglen, H. Bunke, and J. Csirik. Dynamic computation of generalized median strings. Pattern Analysis and Applications, 6(3): 185-193, 2003.

8. X.Jiang, S.Rothaus, K.Rothaus, and D.Mojon. Synthesizing face images by iris replacement: Strabismus simulation. Proc. of Int. Conf. on Computer Vision Theory and Applications, 41-47, Setuba, Portugal, 2006.

9. D. Lopresti and J. Zhou. Using consensus sequence voting to correct OCR errors. Computer Vision and Image Understanding, 67(1): 39-47, 1997.

10. J. Min, M. Powell, and K.W. Bowyer. Automated performance evaluation of range image segmentation algorithms. IEEE Trans. on SMC - Part B, 34(1): 263-271, 2004 .

11. T.B. Sebastian, P.N. Klein, and B.B. Kimia. On aligning curves. IEEE Trans. on Pattern Analysis and Machine Intelligence, 25(1): 116-125, 2003.

12. J.S. Sim and K. Park. The consensus string problem for a metric is NP-complete. Journal of Discrete Algorithms, 2(1), 2001.

13. L. Wang and T. Jiang. On the complexity of multiple sequence alignment. Journal of Computational Biology, 1(4): 337-348, 1994. 